\title{
SP140L is differentially expressed in metastasis to lymph nodes in human breast cancer.
}

Shahan Mamoor, MS ${ }^{1}$

${ }^{1}$ shahanmamoor@gmail.com

East Islip, NY USA

Metastasis to the brain is a clinical problem in patients with breast cancer ${ }^{1-3}$. Between the brain and the breast resides the secondary lymphoid organ, the lymph nodes. We mined published microarray data ${ }^{4,5}$ to compare primary and metastatic tumor transcriptomes for the discovery of genes associated with metastasis to the lymph nodes in human breast cancer. We found that the SP140 nuclear body protein like, encoded by SP140L, was among the genes whose expression was most different in the lymph nodes metastases of patients with metastatic breast cancer as compared to primary tumors of the breast ${ }^{4}$. SP140L was also differentially expressed in the tumor cells of patients with triple negative breast cancer ${ }^{5}$. SP140L mRNA was present at increased quantities in lymph node metastatic tissues as compared to primary tumors of the breast. Importantly, expression of SP140L in primary tumors was significantly correlated with patient overall survival, in lymph node positive patients but not in lymph node negative patients. Modulation of SP140L expression may be relevant to the biology by which tumor cells metastasize from the breast to the lymph node and to the brain in humans with metastatic breast cancer.

Keywords: breast cancer, metastasis, brain metastasis, central nervous system metastasis, lymph node metastasis, the SP140 nuclear body protein like, SP140L, systems biology of breast cancer, targeted 
One report described a 34\% incidence of central nervous system metastases in patients treated with trastuzumab for breast cancer ${ }^{2}$. More recently, the NEfERT-T clinical trial ${ }^{6}$ which compared administration of either neratinib or trastuzumab in conjunction with paclitaxel demonstrated that in a randomized, controlled setting, in breast cancer patients treated with neratinib, not only was the incidence of central nervous system recurrence significantly lower, the time to central nervous system metastasis was significantly delayed as compared to patients administered trastuzumab ${ }^{6}$. The alarmingly high rate of central nervous system metastasis described, as well as data, both anecdotal ${ }^{2}$ and from a randomized, controlled setting ${ }^{6}$ illustrating that treatment with trastuzumab may be associated with these events demands an enhanced understanding of the transcriptional makeup of brain metastatic tissues to support identification of therapeutic targets, whether they are treatment related or not. We performed a global comparative analysis of primary and metastatic tumors in patients with brain metastatic breast cancer ${ }^{4,5}$. We discovered differential and increased expression of the gene encoding the SP140 nuclear body protein like, SP140L, in brain metastatic tissues of patients with metastatic breast cancer.

\section{$\underline{\text { Methods }}$}

We used datasets GSE10893 ${ }^{4}$ and GSE38959 5 for this global differential gene expression analysis of brain metastatic breast cancer in conjunction with GEO2R. GSE10893 was generated using Agilent-011521 Human 1A Microarray G4110A technology with $n=71$ primary breast tumors and $n=7$ lymph node metastases from patients with breast cancer; analysis was performed using platform GPL887. GSE38959 was generated using Agilent-014850 Whole Human Genome Microarray 4x44K G4112F with $n=13$ samples of normal mammary gland ductal cells and $n=30$ samples of primary tumor cells from patients with triple negative breast cancer; analysis was performed using platform GPL4133. The Benjamini and Hochberg method of $p$-value adjustment was used for ranking of differential expression but raw $p$-values were used to assess statistical significance of global differential expression. Log-transformation of data was auto-detected, and the NCBI generated category of platform annotation was used. A statistical test was performed to evaluate whether SP140L gene expression was significantly different between primary tumors of the breast and lymph node metastases in humans with breast cancer using a two-tailed t-test. For Kaplan-Meier survival analysis, we used the Kaplan-Meier plotter online tool $^{7}$ for correlation of SP140L mRNA expression levels with overall survival in $n=726$ lymph node negative breast cancer patients and in $n=452$ lymph node positive breast cancer patients.

\section{$\underline{\text { Results }}$}

We performed global comparative transcriptome analysis of metastatic tumor tissues of patients with metastatic breast cancer using published microarray data ${ }^{4,5}$ to describe the transcriptional landscape of brain metastasis in human breast cancer in an unbiased fashion and for the discovery of novel therapeutic targets.

\section{SP140L is differentially expressed in lymph node metastases of patients with metastatic breast cancer.}

Through blind, systems-level analysis of published microarray data ${ }^{4}$, we identified the SP140 nuclear body protein like, encoded by SP140L, as a differentially expressed gene in the lymph node metastatic tissues of humans with breast cancer (Table 1). When sorting each of the genes expressed in lymph node metastases based on significance of difference as compared to primary tumors of the breast in patients with breast cancer, SP140L ranked 145 out of 18034 total transcripts (Chart 1), equating to 99.2\% differential expression. Differential expression of SP140L in the lymph node metastases of patients with metastatic breast cancer was statistically significant (Chart $1 ; p=1.47 \mathrm{e}-03$ ). 
To attempt to validate differential transcriptome-wide differential expression of SP140L in human breast cancer, we queried a second microarray dataset ${ }^{5}$, here comparing primary tumor cells from patients with triple negative breast cancer to normal mammary gland ductal cells. Again, we identified SP140L as a differentially expressed gene in the tumor tissues of patients with breast cancer (Chart 2). When sorting each of the genes expressed in triple negative breast cancer tumor cells based on significance of difference as compared to normal mammary gland ductal cells, SP140L ranked 12366 out of 45015 total transcripts (Chart 2), equating to $72.5 \%$ differential expression. Differential expression of SP140L in the tumor cells of patients with triple negative breast cancer was statistically significant (Chart 2; $p=2.20 \mathrm{e}-02$ ). Thus, differential expression of SP140L, transcriptome-wide, in the tumor tissues of women with breast cancer was conserved across two independent microarray datasets, both in primary and metastatic tumor tissues.

\section{SP140L is expressed at lower levels in the lymph node metastases of patients with metastatic breast} cancer.

We obtained exact mRNA expression levels for SP140L, in primary tumors of the breast and in brain metastasis of patients with lymph node metastatic breast cancer to determine direction and statistical significance of change in SP140L expression in lymph node metastatic tissues. We observed significantly lower expression of SP140L in the lymph node metastases of patients with breast cancer as compared to primary tumors of the breast: SP140L was expressed at $-0.01 \pm 0.48$ arbitrary units (AU) in primary tumors of the breast, while it was expressed at $0.83 \pm 0.63 \mathrm{AU}$ in lymph node metastatic tissues. The difference in SP140L mRNA levels between primary tumors of the breast and lymph node metastatic tissues was statistically significant (Figure $1 ; p=0.001669$ ).

\section{SP140L expression is significantly correlated with survival outcomes in human breast cancer.}

We performed Kaplan-Meier survival analysis ${ }^{8}$ in $n=726$ lymph node negative patients, and in $n=452$ lymph node positive breast cancer patients, to evaluate whether SP140L tumor expression was correlated with survival outcomes in human breast cancer and to understand whether lymph node status of the patient influenced this correlation. We observed a statistically significant correlation between primary tumor expression of SP140L and overall survival (OS) in patients with lymph node positive breast cancer (Figure 2). Lymph node positive patients whose primary tumors expressed low levels of SP140L possessed median OS of 44.17 months, while lymph node positive patients whose tumors expressed high levels of SP140L possessed median OS of 54.96 months. This difference in OS based on SP140L tumor expression in lymph node positive patients with breast cancer was statistically significant (Figure 2, Chart 3; logrank $p$-value: 0.028; hazard ratio: 0.69 (0.49-0.96)). Primary tumor SP140L expression was not significantly correlated with overall survival in lymph node negative patients (Figure 2, Chart 3; logrank $p$-value: 0.14 ; hazard ratio: 0.77 (0.54-1.09)).

Thus, by mining published microarray data ${ }^{4,5}$ in an unbiased and systematic fashion, we identified the SP140 nuclear body protein like, encoded by SP140L, as among the genes whose expression was most different, transcriptome-wide, in the lymph node metastases of patients with breast cancer, when compared to primary tumors of the breast and in the primary tumor cells of patients with triple negative breast cancer when compared to normal mammary gland ductal cells; we observed significantly decreased expression of SP140L in lymph node metastases as compared to primary tumors of the breast. Further, we found a significant correlation between SP140L expression and patient survival outcomes, in lymph node positive patients but not in lymph node negative patients. 


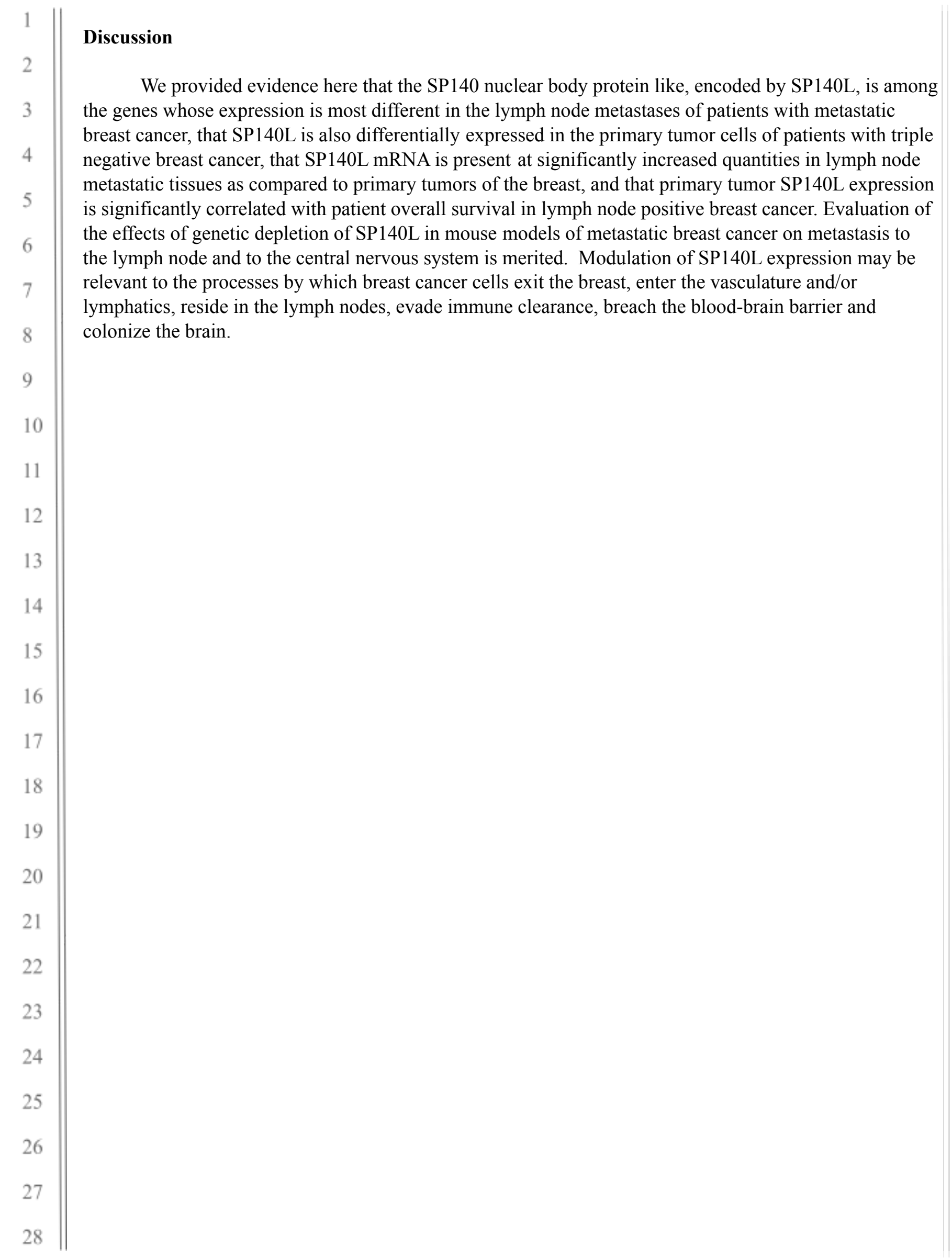




\section{References}

1. Lin, N.U., Amiri-Kordestani, L., Palmieri, D., Liewehr, D.J. and Steeg, P.S., 2013. CNS metastases in breast cancer: old challenge, new frontiers.

2. Bendell, J.C., Domchek, S.M., Burstein, H.J., Harris, L., Younger, J., Kuter, I., Bunnell, C., Rue, M., Gelman, R. and Winer, E., 2003. Central nervous system metastases in women who receive trastuzumab-based therapy for metastatic breast carcinoma. Cancer, 97(12), pp.2972-2977.

3. Tsukada, Y., Fouad, A., Pickren, J.W. and Lane, W.W., 1983. Central nervous system metastasis from breast carcinoma autopsy study. Cancer, 52(12), pp.2349-2354.

4. Weigman, V.J., Chao, H.H., Shabalin, A.A., He, X., Parker, J.S., Nordgard, S.H., Grushko, T., Huo, D., Nwachukwu, C., Nobel, A. and Kristensen, V.N., 2012. Basal-like Breast cancer DNA copy number losses research and treatment, 133(3), pp.865-880.

5. Komatsu, M., Yoshimaru, T., Matsuo, T., Kiyotani, K., Miyoshi, Y., Tanahashi, T., Rokutan, K., Yamaguchi, R., Saito, A., Imoto, S. and Miyano, S., 2013. Molecular features of triple negative breast cancer cells by genome-wide gene expression profiling analysis. International journal of oncology, 42(2), pp.478-506.

6. Awada, A., Colomer, R., Inoue, K., Bondarenko, I., Badwe, R.A., Demetriou, G., Lee, S.C., Mehta, A.O., Kim, S.B., Bachelot, T. and Goswami, C., 2016. Neratinib plus paclitaxel vs trastuzumab plus paclitaxel in previously untreated metastatic ERBB2-positive breast cancer: the NEfERT-T randomized clinical trial. JAMA oncology, 2(12), pp.1557-1564.

7. Györffy, B., Lanczky, A., Eklund, A.C., Denkert, C., Budczies, J., Li, Q. and Szallasi, Z., 2010. An online survival analysis tool to rapidly assess the effect of 22,277 genes on breast cancer prognosis using microarray data of 1,809 patients. Breast cancer research and treatment, 123(3), pp.725-731. 


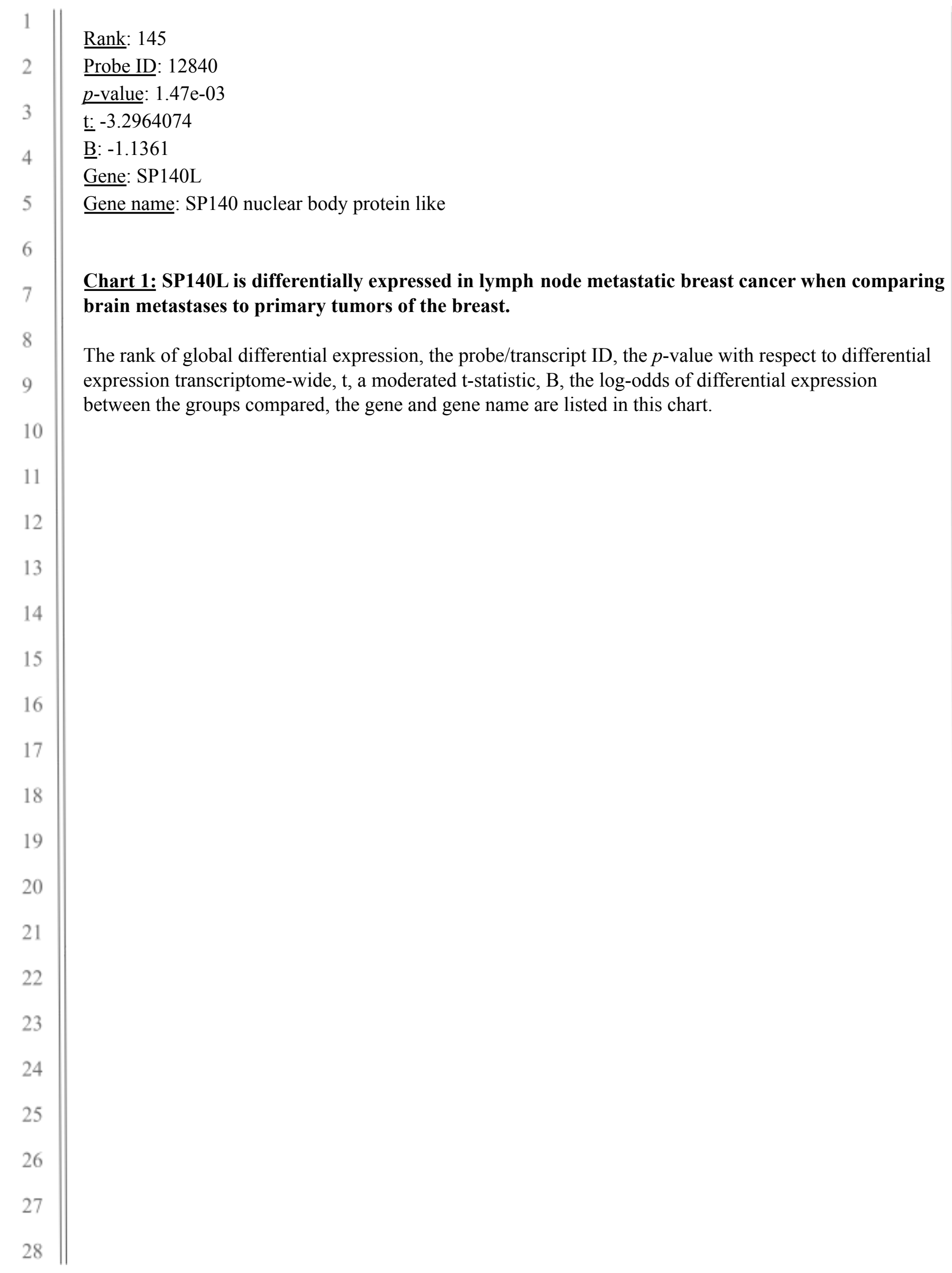




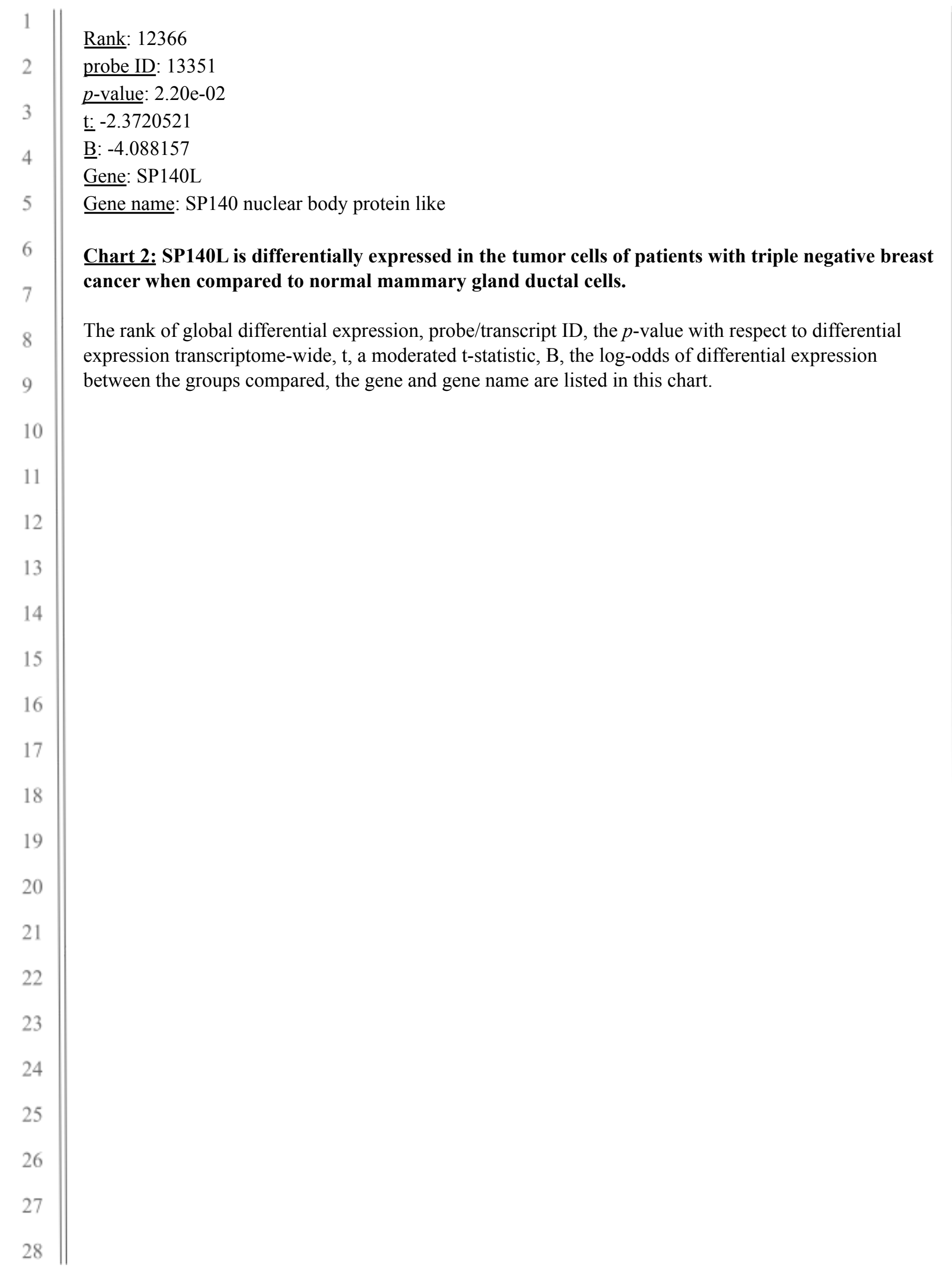




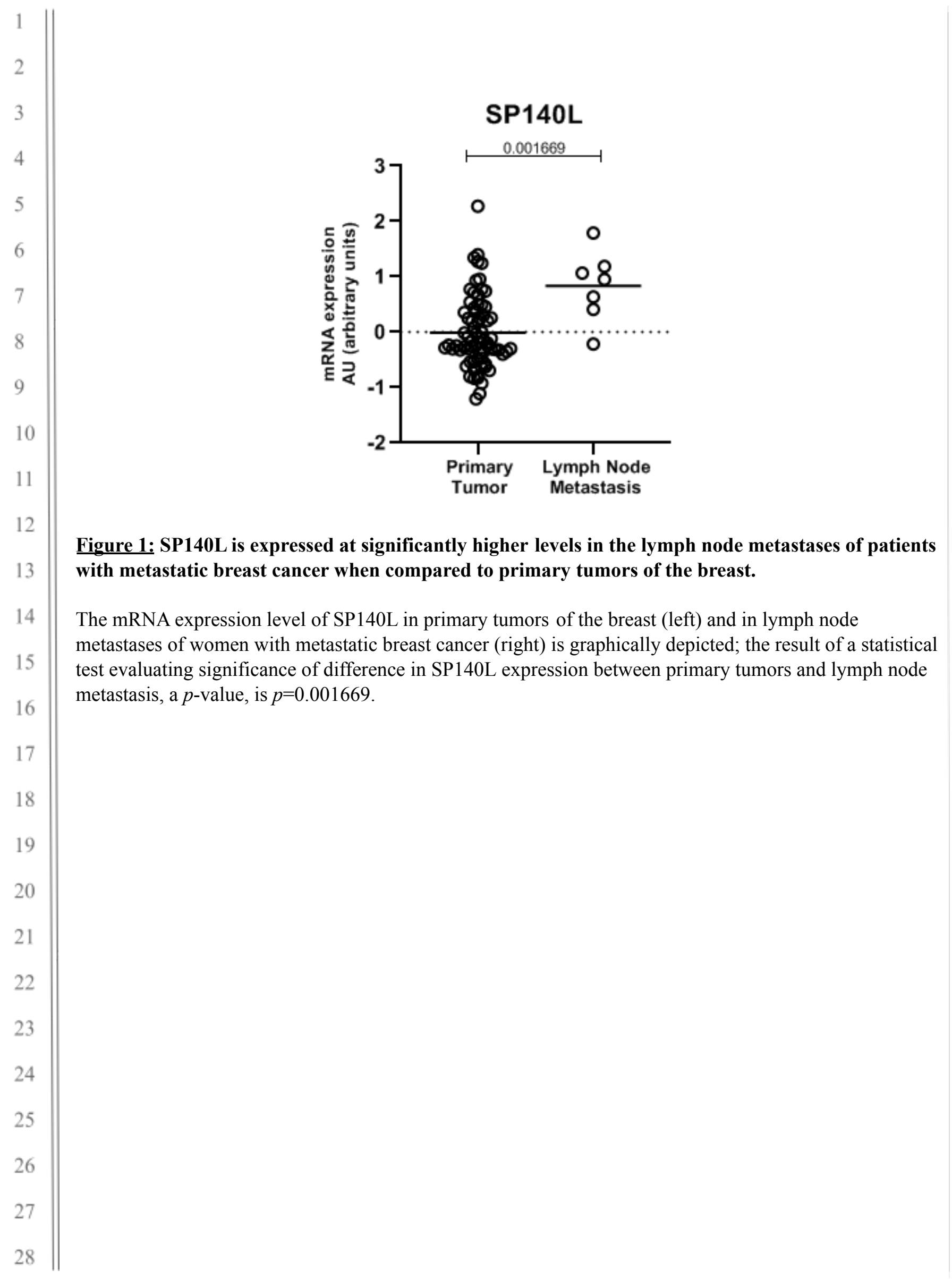



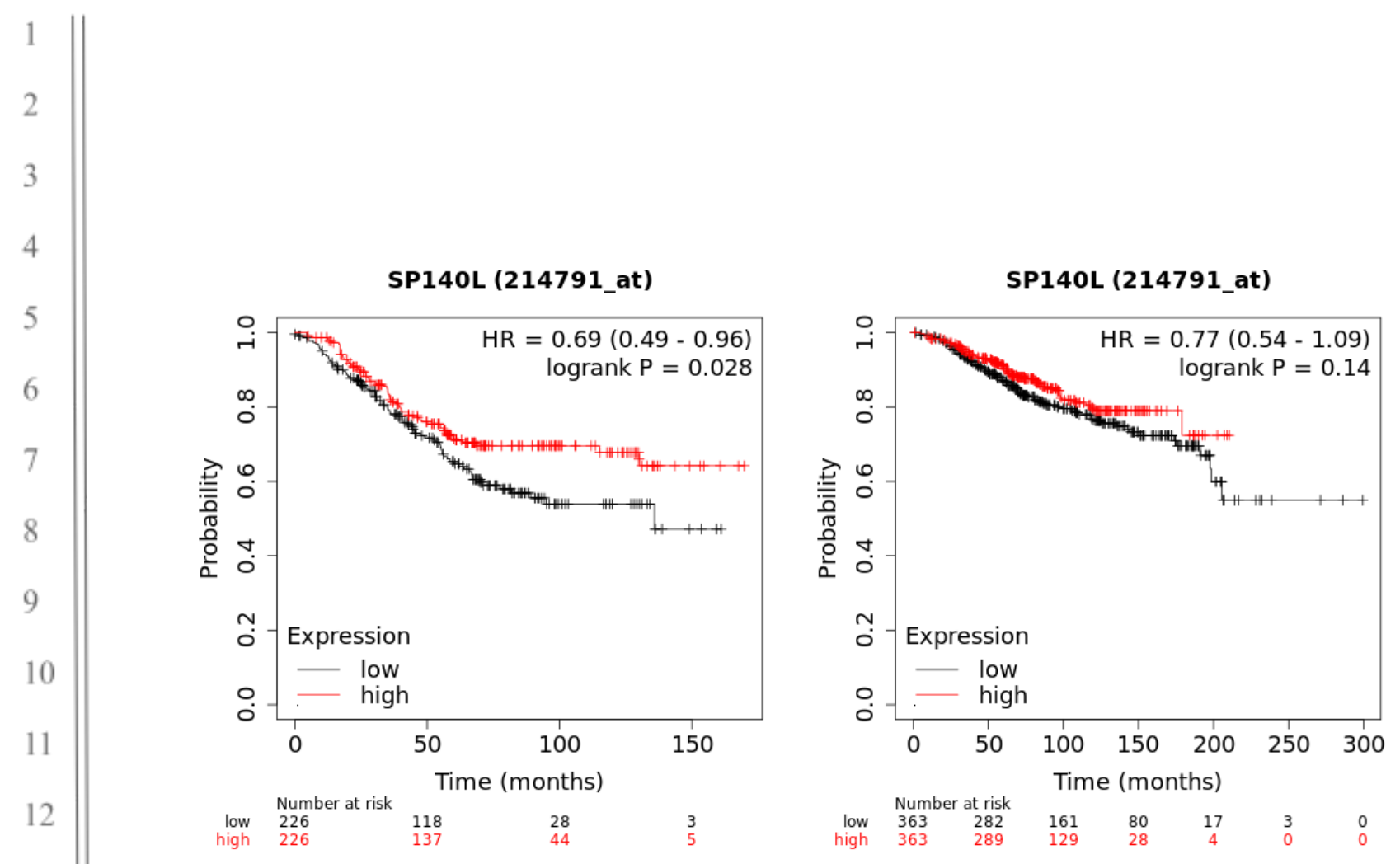

Figure 2: Significant correlation between SP140L primary tumor expression and overall survival in patients with breast cancer, in lymph node positive patients but not in lymph node negative patients.

Depicted in this Kaplan-Meier plot is the probability of overall survival for $n=452$ lymph node positive breast cancer patients and in $n=726$ lymph node negative breast cancer patients, stratified into two groups, based on low or high expression of SP140L in patient primary tumors. The log rank $p$-value denoting statistical significance of difference in overall survival when comparing the two groups, as well as hazard ratio for this comparison is listed above. Listed below is the number of patients at risk (number of patients alive) per interval, after stratification based on SP140L expression; in the first interval, number at risk is number of patients alive; in each subsequent interval, number at risk is the number at risk less those who have expired or are censored. 


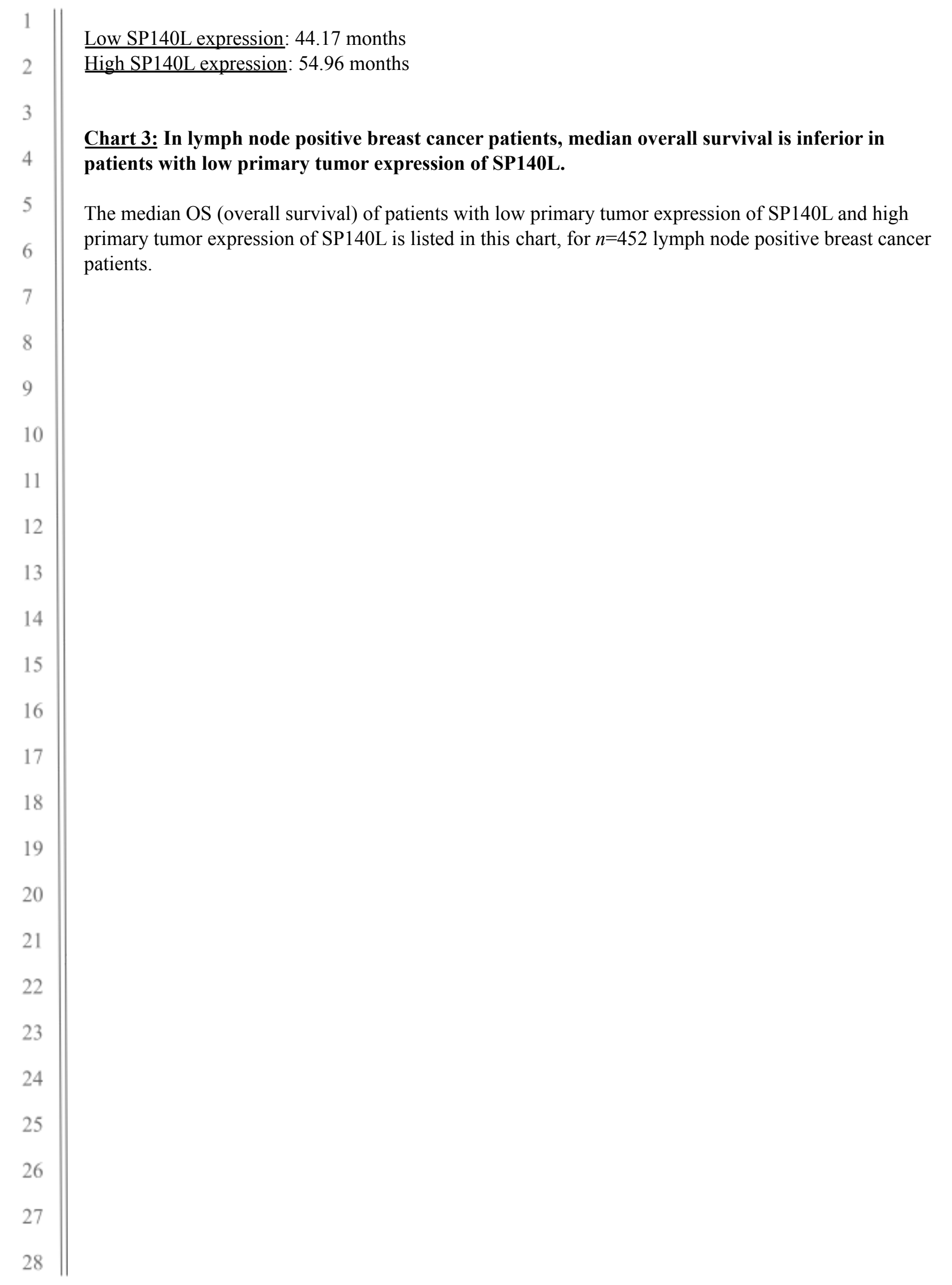

